\title{
The effects of sleep disturbance on school performance: $A$ preliminary investigation of children attending elementary grades
}

\section{Laura Reale}

University of Catania, Italy

\section{Manuela Guarnera}

University of Catania, Italy

\section{Luigi Mazzone}

Children's Hospital Bambino Gesù, Italy

\begin{abstract}
Sleep disorders in children are common. Sleep plays an important role in children's development and sleep disorders can have a substantial impact on their quality of life. Indeed, sleep is crucial for physical growth, behavior, and emotional development and it is also closely related to cognitive functioning, learning and attention, and therefore to school performance. In the present study sleep habits were investigated in 173 schoolage children by using the Children's Sleep Habits Questionnaire (CSHQ). Results show that children attending the third elementary grade had higher CSHQ total score, indicating sleep problems, as compared to other classes. Moreover, an inverse correlation was observed between sleep disturbance and school performance $(p<0.05)$, meaning that children with sleep problems also have a lower performance at school. Our findings imply that sleep disorders could negatively impact school performance.
\end{abstract}

\section{Keywords}

Children's Sleep Habits Questionnaire, CSHQ, decline in school performance, Italy, sleep habits, school-age children

\section{Corresponding author:}

Luigi Mazzone, Child Neuropsychiatry Unit, Department of Neuroscience, IRCCS Children's Hospital Bambino Gesù, Piazza Sant'Onofrio 4, 00165, Rome, Italy.

E-mail: luigi.mazzone@opbg.net 
An increasing number of research studies document the influence of sleep disorders on academic achievement (Millman, 2005; Shin, Kim, Lee, Ahn, \& Joo, 2003; Wolfson \& Carskadon, 2003). Sleep disorders are associated with impairment of memory and cognitive function (Sadeh, Gruber, \& Raviv, 2002) and can negatively affect school performance (Eliasson, Eliasson, King, Gould, \& Eliasson, 2002; Giannotti, Cortesi, Sebastaini, \& Ottaviano, 2002; Perez-Chada et al., 2007) possibly leading to psychological problems including ADHD, depression, and anxiety (Gau et al., 2007, Prehn-Kristensen et al., 2011). Furthermore, sleep could also be important for attention control, executive function, and consolidation of declarative memory. An important role in this context is played by the prefrontal cortex - that is highly susceptible to sleep deprivation and is known to be involved in cognitive function that affects school performance (Curcio, Ferrara, \& De Gennaro, 2006; Durmer \& Dinges, 2005). Indeed, Prehn-Kristensen et al. (2011), based on the fact that the consolidation of declarative memory during sleep is supported by slow oscillations predominantly generated by the prefrontal cortex and that ADHD individuals show lower prefrontal brain activity, proposed that the interplay between the prefrontal cortex and the hippocampus is altered during the first sleep cycle in ADHD individuals. Moreover, it is also well-known that ADHD children and adolescents typically display reduced academic performance and engage in school failure at greater than typical rates (Owens, Stevenson, Hadwin, \& Norgate, 2012). Finally, other studies documented that sleep disorders associated with poor school performance may lead to negative mood, antisocial behavior, and increased the risk of using illegal drugs and alcohol (Ahonen, Nebot, \& Giménez, 2007; O’Brien \& Mindell, 2005).

The data available so far on the link between sleep disorders and school performance arise mainly from samples of children attending middle or high school (Eliasson et al., 2002; Giannotti et al., 2002; Millman, 2005; Perez-Chada et al., 2007; Sadeh et al., 2002; Shin et al., 2003; Wolfson \& Carskadon, 2003) or from clinically referred samples (Wanzek, Jenson, \& Houlihan, 2012) as well as from children who have experienced crises or natural disasters (Widyatmoko, Tan, Seyle, Mayawati, \& Silver, 2011). However, little is known about the possible association between sleep problems and academic achievement in young children. The purpose of our study was to evaluate sleep habits and daytime sleepiness with respect to academic performance in a community sample of children and adolescents attending elementary school in a middle-class, urban setting in Sicily, Italy.

\section{Methods}

\section{Sample}

A total of 154 children (5- to 11-years-old.) were recruited from public elementary schools in a predominantly middle-class urban community in Catania, Sicily, Italy. Students were not significantly different in socioeconomic status. The study was approved by the educational board of the school and by our medical institution. 
Participants' parents completed an informed consent procedure. The sample included the following groups of children according to school level and age: (1) elementary first-grade ( $N=27$, age range: 5- to 6-years-old); (2) elementary second-grade ( $N=38$, age range: 6- to 8-years-old); (3) elementary third-grade ( $N=29$, age range: 8 - to 9-years-old); (4) elementary fourth-grade $(N=29$, age range: 9 - to 10 -years-old); (5) elementary fifth-grade $(N=31$, age range: 10 - to 11-years-old). Data were collected between December 2010 and March 2011.

\section{Assessment}

Children were asked by teachers to give the Children's Sleep Habits Questionnaire (CSHQ) to their parents together with a letter explaining the project and inviting parents to complete the questionnaire. The CSHQ (Owens, Spirito, \& McGuinn, 2000 ) is used to assess sleep patterns and problems in children and consists of 33sleep-disturbance items. Parents are asked to recall the child's sleep behaviors over a 'typical' recent week. Items are rated on a three-point scale (Usually-if the sleep behavior occurred 5 to 7 times per week; Sometimes - for 2 to 4 times per week; and Rarely-for 0 to 1 time per week). The 33-items are conceptually grouped into three subscales: Sleep-disturbance; Night-waking; and Daytime-sleepiness. School performance was defined as high, low or insufficient accomplishment according to parental report, based on the child's final exam of the first four-month period in the 2010 academic year.

\section{Statistical analysis}

Standard descriptive statistics, Pearson's r correlations, and linear regression models were applied to the data in order to evaluate the association between sleep habit variables and school performance. An alpha level of 0.05 was set for statistical significance.

\section{Results}

Data from 154 students (72 boys and 82 females) were collected. No significant differences were detected in age, ethnicity, or gender in our sample from the demographic and sleep variables for the study population. All students included in the study were combined and then divided according to grade grouping. The mean CSHQ total score for children attending the elementary third-grade was higher than for children attending other grades, particularly for the fourth-grade $(20.41 \pm 7.36$ vs. $17.27 \pm 3.82 ; p=0.04)$. The rate of high, low, or insufficient school performance was not different between grades. For the analysis of sleep behaviors by school performance, parent-reports were divided into high and low for each grade level. Consideration of all the students' academic performance showed negative correlation with the CSHQ Total score, indicating that the presence of sleep disorder symptoms increased the risk for a poorer school 
performance $(r=-0.430 ; p=0.018)$. In our sample, the relationship between sleep characteristics and lower school performance was significantly associated with night-waking $(0.53 \pm 0.89$ vs $0.86 \pm 1.04, t=1.9906, p=0.04)$, and daytimesleepiness $(4.61 \pm 2.46$ vs $5.76 \pm 2.67, t=2.5680 ; p=0.01)$.

\section{Discussion}

Several reports have shown that inadequate or disrupted sleep, daytime sleepiness, and poor sleep quality can negatively affect school performance (Blum et al., 1990; Hoffamn \& Steenhof, 1997; Link \& Ancoli-Israel, 1995; Wolfson \& Carskadon, 1998). Our findings support the contention that sleep disturbances negatively affects school performance in a sample of students from first- through fifthgrade. The sleep variables that most affected school performance in school-age children were night-waking and daytime-sleepiness, the latter being significantly associated with difficulty in concentration. Attention problems related to difficulties in concentration during the day are symptoms that may be diagnostic of Attention Deficit/Hyperactivity Disorder, which in turn is often associated with poor school performance (Gruber \& Sadeh, 2004). These findings are in line with several other reports showing that daytime sleepiness is commonly reported as a sleep variable affecting school performance in the school-aged population (Gibson et al., 2006). Another study also reported that daytime sleepiness is a significant sleep variable affecting school performance in high-school students, whereas it did not seem to significantly affect school performance in junior-high students (Pagel \& Kwiatkowski, 2010). Our data are in contrast with this last finding in that, in our sample, sleep-problems, largely attributable to daytime sleepiness, negatively affected school performance also in children attending from first- through fifth-grade.

Our results should be interpreted in the context of several important limitations: (1) The study sample, although representative of the community from which it was drawn, may not necessarily be representative of the general population; (2) even though the community where the study was conducted was quite homogeneous and predominantly middle-class, family socio-economic status was not formally assessed; (3) data collected were limited to parent-report and did not include a comprehensive assessment of other possible sleep habits and behavior; (4) children's cognitive profiles were not evaluated in the present sample in order to avoid confounding by cognitive ability, although this might have provided additional explanations for poor school performance; (5) finally, longitudinal data are necessary to assess the consequences of sleep disorders across the life-span.

A significant correlation was observed between sleep disturbance, as assessed by CHSQ, and poorer school performance in elementary-school students: the prevalence of sleep problems increased significantly with a decline in school performance $(r=-0.430 ; p=0.018)$. Further studies are required to understand whether interventions to modify daytime sleepiness as well as night-waking can improve academic performance in children. School psychologists should be mindful of the 
possibility that children's lack of attention, fatigue, and daytime off-task behaviors may be a function of irregular sleep patterns. Parent-report appears to be an effective way to determine whether children have restful, rejuvenating sleep or engage in night-waking and other forms of sleep disturbances.

\section{References}

Ahonen, E. Q., Nebot, M., \& Giménez, E. (2007). Negative mood states and related factors in a sample of adolescent secondary-school students in Barcelona (Spain). Gaceta Sanitaria, 21, 43-52, doi: 10.1157/13099120.

Blum, D., Kahn, A., Mozin, M., Rebuffat, E., Sottiaux, M., \& Van de Merckt, C. (1990). Relation between chronic insomnia and school failure in pre-adolescents. Sleep Research, 19, 194.

Curcio, G., Ferrara, M., \& De Gennaro, L. (2006). Sleep loss, learning capacity and academic performance. Sleep Medicine Review, 10, 323-337, doi: 10.1016/j.smrv.2005.11.001, doi: 10.1016/j.smrv.2005.11.001.

Durmer, S., \& Dinges, D. F. (2005). Neurocognitive consequences of sleep deprivation. Seminars in Neurology, 25, 117-129, doi: 10.1055/s-2005-867080.

Eliasson, A., King, J., Gould, B., \& Eliasson, A. (2002). Association of sleep and academic performance. Sleep Breath, 6, 45-48, doi: 10.1055/s-2002-23157.

Gau, S. S., Kessler, R. C., Tseng, W. L., Wu, Y. Y., Chiu, Y. N., \& Yeh, C. B., et al (2007). Association between sleep problems and symptoms of attention deficit/hyperactivity disorder in young adults. Sleep, 30, 195-201, doi: 10.1007/s00702-012-0862-3.

Giannotti, F., Cortesi, F., Sebastaini, T., \& Ottaviano, S. (2002). Circadian preference, sleep and daytime behaviour in adolescence. Journal of Sleep Research, 11, 191-199, doi: 10.1046/j.1365-2869.2002.00302.x.

Gibson, E. S., Powles, A. C., Thabane, L., O’Brien, S., Molnar, D. S., Thajanovic, N., \& Chilcott-Tanser, L. (2006). 'Sleepiness' is serious in adolescence: Two surveys of 3235 Canadian students. BMC Public Health, 6, 116, doi: 10.1186/1471-2458-6-116.

Gruber, R., \& Sadeh, A. (2004). Sleep and neurobehavioral functioning in boys with attention deficit/hyperactivity disorder and no reported breathing problems. Sleep, 27, 267-273.

Hoffamn, W., \& Steenhof, L. (1997). Sleep characteristics of Dutch adolescents are related to school performance. Sleep Wake Research in The Netherlands, 8, 51-55.

Link, S., \& Ancoli-Israel, S. (1995). Sleep and the teenager. Sleep Research, 24a, 184.

Millman, R. P. (2005). Excessive sleepiness in adolescents and young adults: Causes, consequences, and treatment strategies. Pediatrics, 115, 1774-1786, doi: 10.1542/peds.20050772.

O'Brien, E. M., \& Mindell, J. A. (2005). Sleep and risk-taking behavior in adolescents. Behavioral Sleep Medicine, 3, 113-133, doi: 10.1207/s15402010bsm0303_1.

Owens, J. A., Spirito, A., \& McGuinn, M. (2000). The Children's Sleep Habits Questionnaire (CSHQ): Psychometric properties of a survey instrument for schoolaged children. Sleep, 23, 1043-1051.

Owens, M., Stevenson, J., Hadwin, J., \& Norgate, R. (2012). Anxiety and depression in academic performance: An exploration of the mediating factors of worry and working memory. School Psychology International, 33(4), 433-449. 
Pagel, J. F., \& Kwiatkowski, C. F. (2010). Sleep complaints affecting school performance at different educational levels. Frontiers in Neuroscience, 1, 1-6, doi: 10.3389/ fneur.2010.00125.

Perez-Chada, D., Perez-Lloret, S., Videla, A. J., Cardinali, D., Bergna, M.A., FernándezAcquier, M., \& Drake, C. (2007). Sleep disordered breathing and daytime sleepiness are associated with poor academic performance in teenagers. A study using the Pediatric Daytime Sleepiness Scale (PDSS). Sleep, 30, 16981703.

Prehn-Kristensen, A., Göder, R., Fischer, J., Wilhelm, I., Seeck-Hirschner, M.Aldenhoff, J. (2011). Reduced sleep-associated consolidation of declarative memory in attention-deficit/hyperactivity disorder. Sleep Medicine, 12, 672-679, doi: 10.1016/j.sleep.2010.10.010.

Sadeh, A., Gruber, R., \& Raviv, A. (2002). Sleep, neurobehavioral functioning, and behavior problems in school-age children. Child Development, 73, 405-417, doi: 10.1111/14678624.00414.

Shin, C., Kim, J., Lee, S., Ahn, Y., \& Joo, S. (2003). Sleep habits, excessive daytime sleepiness and school performance in high school students. Psychiatry and Clinical Neurosciences, 57, 451-453, doi: 10.1046/j.1440-1819.2003.01146.x.

Wanzek, M., Jenson, W. R., \& Houlihan, D. (2012). Recognizing and treating Rett syndrome in schools. School Psychology International, 33(2), 151-166.

Widyatmoko, C. S., Tan, E. T., Seyle, D. C., Mayawati, E. H., \& Silver, R. C. (2011). Coping with natural disasters in Yogyakarta, Indonesia: The psychological state of elementary school children as assessed by their teachers. School Psychology International, 32(5), 484-497.

Wolfson, A. R., \& Carskadon, M. A. (1998). Sleep schedules and daytime functioning in adolescents. Child Development, 69, 875-887, doi: 10.1111/j.14678624.1998.tb06149.x.

Wolfson, A. R., \& Carskadon, M. A. (2003). Understanding adolescents' sleep patterns and school performance: a critical appraisal. Sleep Medicine Review, 7, 491-506, doi: 10. 1053/smrv.2002.0258.

\section{Author biographies}

Laura Reale graduated in medicine and surgery and subsequently completed a doctoral degree program in Child Neurology and Psychiatry at the University of Catania, Italy. She now serves as a Resident in Child Neurology and Psychiatry at the University of Milan, AO San Paolo Hospital.

Manuela Guarnera graduated in psychology at the University of Catania, Italy. She has an extensive background in early childhood developmental theory and cognitive-behavior therapy. Her areas of interest and expertise include individual therapy for children with Autism and ADHD and social difficulties/deficits.

Luigi Mazzone, PhD. After obtaining his doctoral degree, he completed his education with two postdoctoral programs; the first was a postdoctoral visiting fellowship at the Intramural Program of the National Institute of Mental Health (NIMH), Bethesda, MD, USA, followed by the Alexander Bodini Fellowship at the Italian Academy for Advanced Studies in America at Columbia University, 
served at the Paediatric Brain Imaging Laboratory, Columbia University, New York, USA. Dr Mazzone's research focuses on understanding the neurobiological substrates underlying psychiatric disorders such as autism spectrum disorders, ADHD, and Tourette syndrome. He serves as a Clinical Researcher at the Division of Child Psychiatry at Bambino Gesù Hospital in Vatican City, Rome, Italy. 